\title{
Lidil
}

Revue de linguistique et de didactique des langues

47 | 2013

Le verbe pour exprimer le temps

\section{Construction diachronique des usages scolaires du futur périphrastique}

\section{Claudie Péret}

\section{CpenEdition}

\section{Journals}

Édition électronique

URL : http://journals.openedition.org/lidil/3265

DOI : 10.4000/lidil.3265

ISSN : $1960-6052$

\section{Éditeur}

UGA Éditions/Université Grenoble Alpes

\section{Édition imprimée}

Date de publication : 31 mai 2013

Pagination : 61-80

ISBN : $978-2-84310-247-9$

ISSN : 1146-6480

\section{Référence électronique}

Claudie Péret, "Construction diachronique des usages scolaires du futur périphrastique », Lidil [En ligne], 47 | 2013, mis en ligne le 01 décembre 2014, consulté le 01 mai 2019. URL : http:// journals.openedition.org/lidil/3265; DOI : 10.4000/lidil.3265 


\title{
Construction diachronique des usages scolaires du futur périphrastique
}

\author{
Claudie Péret*
}

\begin{abstract}
RÉSUMÉ
Une étude diachronique des grammaires scolaires et des usuels de conjugaison montre comment s'est construit le décalage entre, d'une part, les programmes et manuels actuels et, d'autre part, les usages des élèves. Ce qui a été démontré de façon générale par André Chervel en 1977 est examiné et confirmé en ce qui concerne le point particulier des formes de futur. Depuis le XVIII ${ }^{\mathrm{e}}$ siècle, les ouvrages à destination des élèves affichent une volonté de doter ces derniers de moyens pour maitriser la langue, mais focalisent l'étude, comme dans les grammaires latines dont ils sont les héritiers, sur les parties du discours et non sur les moyens du discours.
\end{abstract}

\begin{abstract}
A diachronic study of school grammar and verb inflection books shows how the gap was built between on the one hand curriculums and schoolbooks and, on the other, pupils' language. André Chervel's 1977 study demonstrated this in general. Here we examine the expression of the future tense. Since the 18th century, schoolbooks have announced that they will endow pupils with means of expression but they focus on parts of speech, not on means of speech, thus mimicking the Latin grammar books they perpetuate.
\end{abstract}

L'étude proposée ici fait suite à celle qui a donné lieu à une communication lors du XI ${ }^{\mathrm{e}}$ colloque de l'AIRDF ${ }^{1}$ (Péret \& Sautot, 2012a). Nous avons mis en évidence que, si une grande partie des performances des élèves sont en adéquation avec l'enseignement prescrit et réalisé, il est possible aussi de constater un écart concernant la morphologie verbale

* EMA Cergy Pontoise.

1. Association internationale pour la recherche en didactique du français. 
du futur. En effet, les élèves ont un usage quasi systématique du futur construit avec aller alors que seul le futur simple est étudié et requis, dans les deux premières années de scolarisation obligatoire (6-7 ans) :

il va ferre venire; on fera du pain (CE1 - 7 ans) $)^{2}$

Les formes complexes étudiées avant la dernière année de scolarisation obligatoire (avant 14 ans) sont celles qui sont construites avec les auxiliaires être et avoir :

après je suis revenue à l'école (CM1 - 9 ans); on aurait joué (6 $6^{\mathrm{e}}$ 11 ans)

Nous appelons forme complexe toute forme verbale construite en plusieurs segments (Cf. Benveniste, 1965, pour l'auxiliation ou BlancheBenveniste, 1984, pour l'appariement d'un verbe auxiliaire et d'un verbe constructeur). Cette définition recouvre celle de périphrase verbale utilisée par Brunot (1922) et Gougenheim (1929) et inclut les formes verbales composées construites avec les auxiliaires avoir et être. La définition suivante que Georges Gougenheim donne de la périphrase verbale dans les premières lignes de son introduction s'applique à ce que nous nommons les formes complexes, puisque la dénomination périphrase verbale n'est utilisée, dans les programmes scolaires du collège, que pour désigner les formes avec constructeur à l'infinitif :

Périphrase verbale : locution formée d'un verbe, en général à un mode personnel, dont le sens propre est plus ou moins effacé, d'une forme nominale, participe ou infinitif, d'un autre verbe qui, lui, a gardé tout son sens. Le $1^{\text {er }}$ sert à indiquer que le procès exprimé par le second est affecté de certains caractères de temps ou d'aspect, de mode, d'action. Le premier élément peut être uni au second soit directement, soit par l'intermédiaire d'une préposition ou d'une locution prépositive. (Gougenheim, 1929, p. 1)

Les élèves utilisent aussi d'autres formes complexes que le futur avec aller, formes abordées lors de la dernière année de scolarisation obligatoire : aller + infinitif, venir de + infinitif, faire + infinitif, laisser + infinitif, devoir + infinitif. Ils utilisent également d'autres auxiliaires, notamment pouvoir + infinitif (Péret \& Sautot, 2012a) :

2. Les exemples sont extraits du corpus Péret \& Sautot (2012). L'orthographe originale est respectée. 
les infirmiers me fesait prendre ( $6^{\mathrm{e}}-11$ ans)

il devra venir de très loin pour aller (CM2 - 10 ans)

il venait de quitter le Royaume ( $5^{\mathrm{e}}-12$ ans)

je pourer venir (CE1 - 7 ans).

Avant que ces formes complexes soient étudiées en fin de scolarité obligatoire, l'accent est mis dans les programmes sur les «temps composés» avec avoir et être, avec une focalisation forte sur l'orthographe du participe passé (Chervel, 1977). Pourtant, le problème des finales verbales en /E/ (Brissaud, Chevrot \& Lefrançois, 2006) réside aussi dans la différence entre infinitif (essentiellement employé dans des formes verbales périphrastiques avec aller, faire) et participe passé employé ou non dans des formes verbales composées avec avoir et être (Péret \& Sautot, 2012a) : 22\% des formes verbales utilisées par les élèves étaient des infinitifs employés avec des auxiliaires alors que $11 \%$ seulement concernaient des participes passés employés avec un auxiliaire (ibid.).

Dans la continuité de ces études, nous cherchons à présent à mieux cerner les origines du décalage observé entre les prescriptions institutionnelles et les usages des élèves en ce qui concerne l'étude du futur formé avec l'auxiliaire aller désormais «futur avec aller».

Les études sur l'usage de l'enfant que nous avons pu consulter sont surtout qualitatives et ne permettent pas d'apprécier la différence entre l'usage des enfants et celui des adultes avec qui ils parlent. Si elles produisent des repères en termes d'acquisition, elles ne permettent pas de conclure quant à l'usage que les enfants ont des marques du futur : futur en aller construit par l'enfant dans un milieu où le futur simple serait majoritairement employé par l'adulte.

Nous avons par ailleurs montré (Péret \& Sautot, 2012b) que l'usage des formes complexes pouvait être considéré comme une étape de l'appropriation du système verbal par les élèves et qu'il est la marque d'une langue «moins académique» que l'on retrouve plus fréquemment à l'oral qu'à l'écrit. Cette langue se caractérise aussi par l'importance d'autres marqueurs que la morphologie verbale pour donner des indications de personne ou de temps : demain + présent; on + base verbale à l'oral pour nous et terminaison -ons :

demain je mange du chocolat (hors corpus)

on n'était allait chez moi pour le lire (CM2 - 10 ans). 
La maitrise d'une variante plus flexionnelle de la langue serait l'apanage des experts, manifestant l'appropriation de ce qui est véhiculé par l'école. Cette gradation entre différents niveaux de langue se retrouve à l'oral en synchronie (Fleury \& Branca-Rosoff, 2010). Elle se retrouve aussi en diachronie : l'évolution du français vers une langue plus analytique est ancienne puisque ses premières manifestations remontent au moyen français (Gougenheim, 1929).

L'hypothèse que nous suivons est d'abord liée à la linguistique historique lors du passage de la description grammaticale du latin à celle du français. La première grammaire scolaire du français (Lhomond, 1780) a ignoré l'usage des locuteurs français et c'est cette grammaire qui a imprimé sa marque à toutes celles qui lui ont succédé (Chervel, 1977), à quelques exceptions près. Pour explorer cette hypothèse, nous nous appuierons sur différentes données qui permettent de constituer un corpus hétérogène, lequel est questionné pour mesurer l'écart entre deux pôles :

- un pôle de l'institution constitué des prescriptions officielles et des grammaires scolaires;

- un pôle des usages et de leur description linguistique.

Ils sont alimentés par différentes sources :

- des descriptions grammaticales du futur, de la grammaire de PortRoyal en 1660, à l'analyse faite en 2010 par Serge Fleury et Sonia Branca-Rosoff du corpus CFPP ${ }^{3}$;

- des présentations du futur dans des ouvrages à destination des élèves, depuis la grammaire scolaire du français ${ }^{4}$ (Lhomond, 1780) jusqu'aux manuels actuels, grâce notamment au travail de recensement effectué par André Chervel en 1977 et à quelques exemples datés du $\mathrm{XVII}^{\mathrm{e}}, \mathrm{XVIII}^{\mathrm{e}}, \mathrm{XIX}^{\mathrm{e}}, \mathrm{xx}^{\mathrm{e}}$ et $\mathrm{XXI}^{\mathrm{e}}$ siècles; un exemple prototypique dans le domaine de la morphologie verbale : de Bescherelle 1851 au Bescherelle 2012;

3. Le corpus de français parlé parisien CFPP 2000 a été constitué à l'initiative de Sonia Branca-Rosoff par trois universitaires de Paris 3, Sonia BrancaRosoff, Serge Fleury et Florence Lefeuvre (équipe SYLED), ainsi que par Mat Pires (équipe LASELDI, Université de Franche-Comté).

4. Nous n'avons pas retenu la grammaire de Restaut (1730) qui aborde explicitement le français pour le latin et propose des déclinaisons du nom. Celle de Lhomond, qui affiche les mêmes objectifs, est moins latinisée et permet une comparaison avec la grammaire latine du même auteur. 
- différentes prescriptions institutionnelles en France concernant le futur: des programmes pour l'instruction publique de 1923 aux programmes pour l'école primaire de 2008;

- des performances d'élèves à l'écrit et à l'oral : corpus écrit de 3500 verbes dont 260 au futur, CFPP 2000;

- des performances d'adultes à l'écrit et à l'oral : corpus des grammairiens, notamment celui de Gougenheim (1929); corpus écrit de commentaires d'articles sur Internet; corpus CFPP 2000.

La confrontation de ces différentes sources permet d'établir la dynamique du maintien d'une prescription qui ne coïncide pas avec les usages. Cette dynamique relève de l'analyse du fonctionnement conjoint de divers aspects sociolinguistiques : le fonctionnement institutionnel, l'analyse opérée par les grammaires descriptives ou prescriptives et les usages. Dans la suite de notre texte, l'accent est mis sur la prescription institutionnelle et les grammaires.

\section{Les premières grammaires du français, modèles toujours actuels}

La première grammaire «scolaire» du français est parue en 1780. D'autres ouvrages l'ont précédée mais à l'usage des non-francophones pour les locuteurs anglais et allemands. C'est là cas notamment de celle de Charles Maupas dont la première édition date de 1607 et à laquelle nous réfèrerons également (nous utiliserons la $3^{e}$ édition, celle de 1632).

À cette époque, la description grammaticale est dominée par ce qu'il est convenu d'appeler la Grammaire de Port-Royal, c'est-à-dire la Grammaire générale et raisonnée (GGR) d'Antoine Arnauld et Pierre Nicole parue en 1660. Cette description a laissé sa marque dans la grammaire française de Charles François Lhomond. La structure générale en est identique, elle-même héritée des grammairiens du Moyen Âge (Donat, $\mathrm{IV}^{\mathrm{e}}$ siècle) : distinction des lettres d'une part et des mots d'autre part, les phénomènes syntaxiques étant abordés lors de l'examen des différentes catégories de mots. Les définitions des catégories sont sémantiques, mais elles sont abordées par leur morphologie et le discours est normatif. Il est intéressant de noter que la Grammaire latine de Charles François Lhomond est construite différemment. Une première partie concerne la description des parties du discours avec une organisation très proche de ce qu'on trouve chez Charles Maupas ou dans la GGR. 
Une deuxième partie concerne la syntaxe de chacune de ces parties et la troisième, intitulée «méthode», est propre à une grammaire du latin pour les Français. On y trouve une description des usages du français en ce qu'ils posent problème dans l'exercice de thème. C'est une façon d'avoir accès à une description du français plus fine que celle de la grammaire du français.

\subsection{Port-Royal : déni d'auxiliarité pour aller}

Dans la GGR, on trouve en germe les confusions qui perdurent dans les programmes scolaires entre les notions de temps, de période temporelle, et de tiroir verbal. Ainsi, les temps simples correspondent à des périodes, la forme j'ay aymé étant considérée comme un temps simple car elle est la traduction d'amavi latin. La notion de temps simple se réfère donc à la simplicité de la pensée en tant que cette forme verbale réfère à une référence temporelle non relative. Cette simplicité de sens et de forme en latin n'a été comprise que sous l'aspect morphologique dans les langues qui lui ont succédé.

Le dernier chapitre de la partie consacrée aux verbes s'intitule: «des Verbes Auxiliaires, des Langues vulgaires». Ce titre associe les formes complexes à l'usage essentiellement oral des langues puisque les langues vulgaires n'ont un statut de langue écrite méritant d'être décrites que depuis quelques décennies.

$\mathrm{Ne}$ sont considérés comme auxiliaires en français que les verbes avoir et être construits avec le participe passé des verbes qu'ils auxilient. N'est donc pas considérée comme forme temporelle la périphrase verbale aller + infinitif.

L'étiquette de «temps composé» de la GGR renvoie à une complexité référentielle dans la mesure où la forme ne se comprend que dans la corrélation temporelle entre deux évènements. La composition est donc temporelle et non formelle. L'analyse scolaire actuelle des temps composés ne réfère certainement pas à cette acception de la composition, mais plutôt à une forme complexe.

Si l'on revient au problème de l'auxiliaire, on constate que dans la GGR, la morphologie des temps est analysée au moyen de la notion de verbes auxiliaires quand cela est nécessaire : «C'est l'usage de certains Verbes qu'on appelle Auxiliaires, parce qu'ils servent aux autres pour former divers temps, avec le participe prétérit de chaque Verbe.» (GGR, 1660, p. 126) La dénomination auxiliaire est donc réservée aux formes complexes construites avec le participe de l'auxilié. 
Les relations d'auxiliation sont envisagées à peu près comme l'a fait Benveniste (1965) pour deux des cas envisagés par ce dernier. Il s'agit des auxiliations de temporalité et de diathèse (passif). L'auxiliation de modalité est absente. Cette absence est d'autant plus intéressante que les auteurs de la GGR ont analysé des formes complexes autres que marquant les temps composés de «l'affirmatif». Ils indiquent en effet :

Il y en a deux [des auxiliaires] qui sont communs à toutes les Langues, estre \& avoir. Quelques-unes en ont encore d'autres, comme les Allemans Werden, devenir, ou Wollen, vouloir, dont le présent estant joint à l'infinitif de chaque Verbe en fait le futur. Mais il suffira de parler des deux principaux estre \& avoir. (GGR, p. 126)

On constate donc un «déni» d'auxiliarité pour le verbe aller et le déni d'une forme périphrastique pour le futur français. Or ce verbe est attesté avec valeur d'auxiliaire du futur dès le XIV siècle (Gougenheim, $1929)^{5}$. La grammaire de Charles Maupas, à destination des allophones, prend plus en considération les formes périphrastiques. De nombreux auxiliaires, définis comme tels, sont analysés dans leur construction et leur sémantisme. Il s'agit des auxiliaires que la grammaire traditionnelle nomme «modaux ». Il est intéressant de noter qu'une remarque est faite sur les différentes significations liées à la syntaxe de venir : «je viens de»+ infinitif, «je viens à»+ infinitif, «je viens»+ infinitif sans que le sémantisme ne soit explicité. Le verbe je vais est présent dans la liste des verbes qui régissent directement un autre infinitif. Ces verbes «signifient volonté, pensée ou permission». Le verbe aller est reconnu comme «auxiliaire» mais non de temporalité. Par ailleurs, chez Charles Maupas, dont l'objectif était aussi de bien faire prononcer le français aux étrangers, on trouve une remarque intéressante concernant la prononciation du $r$ final de l'infinitif :

Je trouve niaise, la fantasie d'aucuns, qui affectent une lasche prononciation du bas populas, d'obmettre et supprimer du tout, toutes les $r$, finales, ainsi. Vous plaist-il venir disné avec imoy, vous me ferez plaisi au lieu de dire. Venir, disner, plaisir avec modérée prononciation de l'r. (Maupas, 1632, p. 21)

5. Gougenheim ne cite que peu d'exemples et précise que la valeur de futur qu'il nomme «prochain» s'est construite parallèlement à celle de futur inchoatif attesté également dès l'ancien français. 
La finale verbale se prononçant pour l'infinitif, il n'y avait pas de problème orthographique sur ces finales et leur contexte d'emploi.

La référence de toutes ces grammaires est celle du latin. En latin, $e o$ (je vais) n'est considéré que comme verbe de mouvement, quoiqu'un exemple à valeur de futur ait été trouvé chez Cicéron (Gougenheim, 1929). La description grammaticale du français n'a pu s'affranchir de la langue latine et de sa description.

Nous avons donc analysé les grammaires de Charles François Lhomond, pour mettre en évidence la marque de ce lien.

\subsection{Lhomond, l'affirmation d'une focalisation sur la langue latine}

En 1779, Charles François Lhomond publie les Éléments de la grammaire latine, puis, en 1780, les Éléments de la grammaire française. Ce dernier ouvrage a été réédité puis remanié par des continuateurs, mais on en retrouve, un siècle plus tard, au moment où Louis-Nicolas Bescherelle publie sa Grammaire pour tous, les caractéristiques en ce qui concerne la syntaxe et la morphologie verbale.

Il nous a paru important de rappeler ici l'objectif que s'est donné Charles François Lhomond. Dans la préface de la première édition, il précise :

Les enfants comprennent plus aisément les principes de la grammaire, quand ils les voient appliqués à une langue qu'ils entendent déjà, et cette connaissance leur sert comme d'introduction aux langues anciennes qu'on veut leur enseigner. Nous avons de bonnes grammaires françaises mais je doute que l'on puisse porter un jugement aussi favorable des abrégés qui ont été faits pour les commençants. Les premiers éléments ne sauraient être trop simplifiés. (Lhomond, 1779)

Une grammaire de la langue française pour les commençants, donc, mais qui est un tremplin pour l'apprentissage des langues anciennes. C'est ce qui justifie une terminologie empruntée au latin.

Le verbe, dans l'ouvrage de 1780, page 20, est défini exactement comme dans le Bescherelle de 1834 : «Le verbe est un mot dont on se sert pour exprimer que l'on est ou que l'on fait quelque chose.» Cette définition sémantique est aussi celle que l'on trouve dans la $2^{\mathrm{e}}$ édition de la grammaire latine. Elle est complétée, pour la grammaire du français, par une marque de désagglutination dans le passage du latin au français, rendant nécessaire la présence du pronom de conjugaison, la désinence verbale étant insuffisante pour discriminer la personne : «On connoît un verbe en français quand on peut y ajouter ces pronoms, je, 
tu, il, nous, vous, ils ${ }^{6} . »$ On voit bien ici l'application de la connaissance de la grammaire du français pour aborder celle du latin. Dans les tableaux de conjugaison du français, le pronom sujet apparait, ainsi que la conjonction que devant les formes de subjonctif alors qu'aucun subordonnant n'apparait dans les tableaux du latin. Ces ajouts figurent déjà dans la traduction que Charles François Lhomond propose dans la grammaire du latin. Par ailleurs l'auteur juge nécessaire de donner une définition du prétérit défini (passé simple), et du prétérit indéfini (passé composé) :

On appelle prétérit défini celui qui marque un temps entièrement passé; exemple : j'eus hier la fièvre. On appelle prétérit indéfini celui qui marque un temps dont il peut rester encore quelque partie à s'écouler; exemple : j'ai eu la fièvre aujourd'hui.

Concernant les auxiliaires, Charles François Lhomond (1780, p. 22) dit qu' «il y a deux verbes que l'on nomme auxiliaires, parce qu'ils aident à conjuguer tous les autres : nous commencerons par ces deux verbes ». Et de commencer avec avoir. Rien n'est dit sur les formes verbales complexes.

Il faut consulter la «méthode» de la grammaire latine pour avoir en filigrane une description de ces formes complexes. Elles apparaissent sous l'étiquette «locutions françaises» qu'il faut «tourner» pour pouvoir les traduire :

Faites-moi savoir, doit se «tourner» en faites en sorte que je sache, fac ut sciam.

Il le fit tuer, c'est-à-dire il ordonna qu'il fût tué, jussit eum occidi.

Venir de..., devant un infinitif, se tourne par tout à l'heure, modò advenit. (Lhomond 1850, p. 229)

Ainsi sont passés en revue les différents auxiliaires d'aspect, de temps, de modalité ou de diathèse sans qu'une analyse en ait été faite. Et aller + infinitif n'est pas considéré comme «locution française».

Charles François Lhomond a donc institué une grammaire française attribuant au français des caractères de la langue latine, évacuant des tournures qu'il appelle pourtant «gallicismes» dans sa grammaire latine. De ces gallicismes est écartée la forme complexe aller + infinitif pourtant attestée dans la littérature de cette époque. Et c'est cette grammaire

6. Il est à noter que cette définition distributionnelle est très utilisée par les élèves actuellement (Cf. Gourdet). 
qui a servi de modèle pour la tradition grammaticale (notamment les tableaux de conjugaison).

\subsection{Bescherelle, l'inscription dans la durée ou la sédimentation d'une référence}

De la Grammaire nationale de 1834 (rééditée jusqu'en 1877) au Manuel de conjugaison qui porte le nom des premiers auteurs, le nom de Bescherelle renvoie maintenant au prototype des usuels pour le verbe, mais aussi, dans une moindre mesure, pour la grammaire et l'orthographe.

Peu de temps après la parution de la Grammaire nationale, les frères Bescherelle, Louis-Nicolas dit ainé et Henri, dit jeune, ont fait paraitre la Grammaire des épiciers ( $2^{\mathrm{e}}$ édition 1939) dans laquelle sont présentées les «fautes» de prononciation et de construction repérées comme telles par les deux grammairiens. Rien n'est dit sur la prononciation des finales verbales en /E/ ni sur les constructions périphrastiques. L'homophonie de l'infinitif et du participe passé est devenue la norme sans que les auteurs, soucieux de l'hétérographie des participes passés, la prennent en compte. Le futur avec aller, attesté à cette époque, n'est pas identifié comme fautif, mais n'est pas pour autant décrit dans les grammaires à destination des élèves et des étrangers puisque ce sont les mots et non les besoins linguistiques qui structurent ces ouvrages.

Louis-Nicolas Bescherelle a produit des ouvrages de vulgarisation au moment où la loi Guizot concernant les écoles primaires commençait à s'appliquer. Saint-Gérand (2000) a montré la contribution des travaux des frères Bescherelle à la construction d'un français de référence normé.

Le sous-titre du Manuel usuel et populaire dont nous utiliserons la $2^{\mathrm{e}}$ édition (1851) revue, augmentée et corrigée, est significatif :

À l'aide duquel les enfants, les classes ouvrières, les gens de la ville et de la campagne, les étrangers peuvent apprendre seuls, sans ennui, sans fatigue et en peu de temps, l'art le plus indispensable, c'est-à-dire l'art de parler, de lire et d'orthographier la langue française.

La lecture du manuel permet de comprendre comment s'est faite la filiation entre les premières grammaires et celles que nous connaissons actuellement. Il est considéré par l'auteur comme le premier ouvrage de ce genre.

Une première partie est consacrée aux «simples règles d'orthographe» et à la «grammaire élémentaire», une deuxième partie à la prononciation. 
Dans la «grammaire élémentaire», on retrouve les tableaux de conjugaisons tels qu'ils étaient dans les ouvrages de Charles François Lhomond. C'est le verbe être dont la morphologie est donnée en premier. Chaque forme de ce verbe est accompagnée d'un adjectif, ce qui place bien le verbe être comme étant le plus fréquent, mais dans sa valeur attributive et non comme auxiliaire. Vient ensuite avoir. Rien n'est dit des formes périphrastiques. Les verbes sont regroupés en quatre groupes qui se veulent un calque des quatre conjugaisons du verbe latin. De nombreuses pages sont consacrées à ce qui est appelé la «syntaxe du participe», mais il ne s'agit que d'étudier les différentes places du participe passé pour en donner les règles d'orthographe. L'infinitif ne posant pas de problème d'orthographe, il ne bénéficie pas d'un chapitre consacré à sa «syntaxe». La syntaxe du verbe concerne essentiellement l'accord avec le sujet, les constructions directes ou indirectes, la concordance des temps avec le subjonctif. Les développements consacrés à la syntaxe du verbe sont avant tout des «leçons» d'orthographe puisque la connaissance des constructions directes et indirectes a un impact sur l'orthographe du participe passé.

L'absence du futur en aller dans le manuel n'est donc pas due à un usage marginal ou populaire, mais à une absence historique de nécessité d'explicitation orthographique dans les ouvrages qui lui ont servi de modèle. Ces conclusions renforcent celles d'André Chervel dans son analyse de la grammaire comme étant au service de l'orthographe.

L'édition de 1884 concentre les éléments de la Grammaire pour tous et du Dictionnaire général et usuel de tous les verbes français. L'ouvrage a alors l'organisation que nous lui connaissons actuellement.

C'est avec l'édition de 1950 que le nom de Bescherelle passe d'un nom d'auteur à un titre d'ouvrage de référence. Cette édition, assumée cette année-là par la libraire Joseph Gibert à Paris, prend en compte les modifications dans la terminologie grammaticale apportée par la Grammaire de l'Académie française, parue en 1932. Comme dans cette grammaire, c'est le verbe avoir qui est présenté en premier et les verbes sont présentés en trois groupes.

Édité depuis 1966 chez Hatier, le manuel connait des ajustements successifs. Il faudra attendre l'édition de 1999 pour que le verbe être soit de nouveau donné en premier, présenté comme auxiliaire et qu'apparaisse, dans la partie «syntaxe du verbe», une présentation des «semiauxiliaires». Aller est décrit comme semi-auxiliaire servant à construire une périphrase verbale de futur proche. En 2012, sur le site de l'éditeur, un slogan «la langue française évolue, Bescherelle aussi !» laisse 
espérer une présentation de la syntaxe du verbe différente. Il n'en est rien. Il s'agit de présenter de nouveaux ouvrages, déclinant l'ancienne structure en différents niveaux de scolarité. L'édition 2012 «entièrement revue» n'apporte pas de changement significatif. Il faut signaler une nouveauté cependant : les responsables scientifiques de l'édition sont nommés. Pendant plus d'un siècle et demi, l'ouvrage est devenu une institution qui a servi et sert toujours de modèle à d'autres usuels. Les éditions successives se sont déposées dans le savoir partagé du français, voire la noosphère pour faire d'un fossile, la langue morte qu'est le latin, la référence pour écrire le français actuel. Une maison d'édition prendrait-elle le risque de proposer un manuel à destination des élèves qui contredirait cette doxa? Les éditeurs actuels du Bescherelle pourraient-ils eux aussi proposer une approche radicalement différente?

\section{L'école, lieu de tensions entre tradition grammaticale et langue actuelle}

\subsection{Les difficultés des politiques publiques en matière d'enseignement}

Quelle peut être la position de l'école dans cette situation? Les programmes pour l'école sont le fait de politiques publiques et sont issus d'un processus long de consultation de différentes instances (Carlotti, 2012) qui partent d'un constat et de l'analyse de ce constat, et doivent pour proposer des actions tenir compte des contraintes liées à la compréhension par le public de la politique voulue et de pressions socioéconomiques. En élargissant le cadre étroit du point de langue qui nous intéresse au programme de l'enseignement grammatical en général, examinons les problèmes rencontrés par les politiques pour l'école face au constat établi.

Le constat est partagé par les gouvernements qui se sont succédé dernièrement. Tout en restant prudent dans l'interprétation des chiffres puisqu'il est difficile de comparer des résultats d'enquêtes qui ne suivent pas le même protocole (enquêtes de l'Agence nationale de lutte contre l'illettrisme, enquêtes internationales, évaluations nationales, enquêtes de chercheurs ${ }^{7} .$. ), on peut avancer que le pourcentage d'élèves quit-

7. Enquête INSEE «Information et vie quotidienne 2011-2012», enquêtes PISA et OCDE, évaluations nationales de l'Éducation nationale française... 
tant l'école en situation d'illettrisme ou de maitrise insuffisante de la langue écrite n'arrive pas à décroitre malgré les différentes mesures prises. Une analyse rapide reporte sur l'école la responsabilité de cet échec. L'analyse que nous venons de faire de l'écart entre les grammaires pour les élèves et la langue qu'ils utilisent peut expliquer cet échec. L'enfant apprenant le français devrait apprendre en même temps cette doxa qu'il découvre à l'école comme devant être une évidence. Pour arriver à freiner l'élargissement du fossé entre discours véhiculé par l'école (en contradiction avec les recherches en linguistique) et la langue parlée par les élèves, il faudrait que l'enseignement de la grammaire devienne tout autre : non pas un discours archaïque focalisé sur l'orthographe et notamment celle du participe passé, mais une découverte des moyens linguistiques que la langue propose pour exprimer sa pensée et comprendre celle de l'autre. On pourrait ainsi aborder, en prenant l'exemple qui nous préoccupe, les différentes possibilités de situer un énoncé dans le futur : présent + indicateur temporel (usage très fréquent à l'oral y compris par les enseignants); futur avec aller... et, tout comme le passé simple enseigné comme un temps de l'écrit qui tend à disparaitre, le futur simple. Cela suppose une révolution dans les méthodes employées en classe, un heurt avec l'image de l'école que les parents ont en eux (image qui ne correspond pas forcément à la scolarité qu'ils ont suivie), transformation en profondeur des ouvrages de vulgarisation sur la langue. Ces difficultés sont connues de l'analyse des politiques publiques et expliquent les réformes par petits pas, l'incrémentalisme, tel qu'il a été décrit initialement par Lindblom (1959). Pourtant quelques tentatives de pas plus grands sont à signaler.

\subsection{Deux tentatives de franchissement du fossé}

La première tentative est celle du «plan Rouchette» paru dans les années qui ont suivi les «révolutions » structuraliste, générativiste et énonciative de la linguistique. Ce plan précisait bien cette posture :

Les progrès de la linguistique conduisent à considérer comme nécessaire l'abandon de quelques principes généraux antérieurement admis. C'est ainsi que certaines pratiques pédagogiques traditionnelles se fondent sur l'idée que la maîtrise de la langue part de la réflexion pour aboutir à l'usage. Nous pourrions citer maints exemples [...] : dans un enseignement grammatical qui, faisant construire les fonctions à partir des natures, passant uniformément de la phrase simple (proposition indépendante) à la phrase complexe, cherche à améliorer l'expression en s'appuyant presque exclusivement sur une réflexion abstraite et non sur 
le fonctionnement réel de la langue. Or, depuis un quart de siècle, la linguistique a montré qu'observer ce fonctionnement est la condition préalable et nécessaire de toute réflexion féconde et de tout perfectionnement. (Rouchette, 1971, p. 4)

Cette période est celle du premier ouvrage d'application didactique de la recherche en linguistique : en 1970, au moment de l'élaboration du plan Rouchette, Émile Genouvrier et Jean Peytard font paraitre Linguistique et enseignement du français. Dans leur chapitre consacré au «français fondamental», ils abordent les différences entre le français parlé décrit par Jean Dubois dans la Grammaire Larousse du français contemporain (GLFC) et celui qui est enseigné traditionnellement :

Un nouveau temps verbal est maintenant enregistré dans les tableaux de conjugaison (v. GLFC), le «futur périphrastique» (je vais chanter), en face du «futur simple» (je chanterai); les relevés statistiques montrent, en effet, que la langue emploie un futur périphrastique pour deux futurs simples. (Genouvrier \& Peytard, 1970, p. 27)

Ces apports à la réflexion didactique auront une faible répercussion dans l'édition scolaire. Émile Genouvrier et Claudine Gruwez dirigeront chez Larousse une collection de manuels intitulée «Structure de la langue française». Dans le manuel Grammaire nouvelle pour le CE1, paru en 1972, sont proposées les deux formes de futur, le futur simple appelé «futur 1 » et le futur avec aller, futur 2. C'est en décembre de cette même année que paraissent les Instructions pour l'enseignement du français l'école élémentaire, influencées par le plan Rouchette. L'accent est mis sur la reconnaissance de la spontanéité de l'enfant qu'il faut pouvoir prendre en compte. La démarche s'appuie ensuite essentiellement sur les textes lus. Dans la partie conjugaison, les auteurs des instructions déplorent la maitrise insuffisante de la morphologie verbale et l'imputent à la seule homophonie de formes hétérographes. Il n'est nullement fait allusion au futur périphrastique dans la liste des savoirs à acquérir. Cette absence explique que le «futur 2 » ne se retrouve plus dans les éditions scolaires des années suivantes.

La deuxième tentative, plus récente n'a pas connu une fortune meilleure. Il s'agit des programmes pour l'école de 2002. Lors de leur élaboration, comme pour les programmes précédents, il a été tenu compte des résultats de la recherche en linguistique, en psychologie cognitive, en didactique ainsi que d'une consultation des acteurs de terrain. Le souhait déjà affirmé de partir de l'expérience des élèves a été réaffirmé. En ce qui concerne la grammaire, pour éviter que se reproduisent encore 
les anciennes pratiques, l'accent a été mis sur la démarche dans l'intitulé même de cette partie du programme qui s'est appelée «Observation réfléchie de la langue». Le pas était sans doute trop grand. Malgré l'effort de formation continue qui a accompagné la mise en œuvre de ces programmes, le sentiment de «perdre la grammaire»a été très fort tant dans les familles des élèves que chez les enseignants. La difficulté et la crainte de pratiquer différemment l'enseignement grammatical a provoqué des réactions de repli sur les anciennes pratiques (Péret, Sautot \& Brissaud, 2008; Élalouf \& Péret, 2009). Ces nouveaux programmes ont été suivis de peu par la modification de la formation initiale des enseignants. La corrélation de ces deux réformes a laissé peu de place à la construction d'une pratique professionnelle nouvelle. C'est sans doute le cumul de deux réformes qui a fait que les programmes de 2002 n'ont pas produit les effets escomptés. Un pas en arrière, plus grand que celui qui avait été fait en avant en 2002, a été fait par les programmes 2007 puis 2008. Le moyen de faire reculer le nombre d'élèves en échec s'est traduit non par une proposition de méthode différente, mais par une programmation plus importante de «savoirs de base» à enseigner à l'école élémentaire.

\section{Quelles solutions pour résoudre les tensions?}

Il n'est pas dans l'intérêt des chercheurs en didactique de proposer «le pont de la rivière Kwaï» de l'enseignement grammatical. Il serait voué à la destruction. Quelles propositions épistémologiques permettrait une rénovation de l'enseignement grammatical? Une première étape pourrait consister à ne pas imposer l'analyse grammaticale trop tôt dans la scolarité des élèves. L'analyse linguistique pourrait ne commencer qu'au lycée. Le socle commun de connaissances et de compétences de 2006 stipule qu'à l'issue de la scolarité obligatoire, les élèves doivent «maitriser suffisamment les outils de la langue que sont le vocabulaire, la grammaire et l'orthographe». Maitriser suffisamment les outils de la langue ne signifie pas être capable d'en faire l'analyse. De nombreux étudiants arrivent en master et se préparent au métier d'enseignant alors qu'ils ne savent pas attribuer le métaterme adéquat pour désigner un complément d'objet indirect ou un complément circonstanciel. Cela ne les a pas empêchés de lire et de s'exprimer correctement et d'atteindre un niveau universitaire; tout comme de nombreux salariés, voire entrepreneurs et responsables peuvent avoir une langue très correcte sans pour autant connaitre les délices de l'analyse logique et grammaticale. 
À l'école élémentaire, une observation et une appropriation de ces outils de la langue est largement suffisante. L'observation réfléchie pourrait être l'objet du collège et l'analyse du lycée. Reste le problème de la terminologie. Il n'est dans l'intérêt de personne d'instaurer une rupture entre les locuteurs français et leur école. Si la grammaire désigne le domaine dans lequel s'effectueront les observations, les manipulations et l'analyse, il est logique que ces activités s'appellent «grammaire» des premières années du primaire au lycée tout comme s'appellent «mathématiques» des activités de dénombrement aussi bien que l'analyse algébrique. Nous avons utilisé ici le conditionnel. Cette proposition serait sans doute trop novatrice présentée ainsi, comme l'ont prouvé des réactions d'enseignants et de chercheurs. Elle suppose que lui précèdent des instructions présentant ce que peuvent être des activités grammaticales préparatoires à l'analyse : classer les moyens linguistiques permettant de situer un énoncé dans le futur et déterminer des sous-classes selon le procédé utilisé. Il s'agit là de moyens nécessaires à l'analyse, avant que la terminologie et la définition logique ne soient imposées.

Si on observe que l'usage pour situer un énoncé dans le futur recourt à trois moyens linguistiques, il faudra bien nommer le futur en aller qui connait une crise identitaire. Après un déni d'existence, il a connu plusieurs noms : futur prochain (Gougenheim, 1929) ${ }^{8}$; futur immédiat ${ }^{9}$; ultérieur (Damourette \& Pichon, 1911-1936); futur périphrastique (Brunot, 1922); futur aller noté fut pres (Gross, 1986); futur en va (à côté du futur en -ra, Jeanjean, 1988); futur 2 (Genouvrier \& Gruwez, 1972)... Cette dernière dénomination qui est celle choisie par une édition scolaire présentait l'inconvénient de ne pas être «lisible». Quel était le futur 1, quel était le futur 2? Les élèves admettent qu'un passé composé est un tiroir verbal constitué de deux éléments. La logique voudrait que l'appellation composé s'applique à toutes les formes verbales avec auxi-

8. Gougenheim propose l'appellation «futur prochain» en prenant comme argument les exemples trouvés dans la littérature depuis la fin du Moyen Âge. L'immédiateté est prouvée par le contexte, notamment la présence d'adverbes comme bientôt, prochainement. Si les auteurs ont eu recours à ces adverbes, c'est bien parce que la forme verbale n'a pas, en elle-même, la valeur d'immédiateté. Cette appellation a pourtant été reprise de nombreuses fois par la suite.

9. Cette appellation est aussi utilisée ainsi que la deuxième personne du présent de l'impératif dans les méthodes de français langue étrangère. 
liaire. Le futur en aller ne peut pourtant pas s'appeler futur composé, l'appellation convenant sans doute mieux au futur antérieur. La difficulté à prononcer et à orthographier périphrastique ne fait pas de cette appellation un bon candidat pour l'école élémentaire. La désignation utilisée dans cet article présente elle aussi un inconvénient : pourquoi désignerait-on un futur par l'auxiliaire qui sert à le construire alors que le passé n'est pas désigné par l'auxiliaire? Certes, il y a deux auxiliaires pour les temps du passé. Et pourquoi seulement futur avec aller alors que d'autres auxiliaires, beaucoup plus rares, il est vrai, servent aussi à construire un futur : penser, s'apprêter à... Un des petits pas qui pourrait être fait sur le chemin de la rénovation de l'enseignement grammatical serait de faire figurer ce futur avec un nom issu ou non de toutes les études citées dans cet article et de l'insérer dans une terminologie grammaticale «officielle». La partie grammaire du verbe des usuels de conjugaison pourrait ainsi s'en emparer. Et si c'est dans le Bescherelle, alors... Les éditions à destination des étrangers ne s'embarrassent pas de la tradition grammaticale et c'est le futur avec aller qui est enseigné en premier. Mais il faut dire que l'entrée dans les savoirs grammaticaux se fait par les besoins langagiers.

Cette évolution à pas mesurés devrait s'accompagner d'une formation des enseignants à la pratique de l'observation de la langue. Cette formation serait d'autant mieux acceptée que la charge des programmes serait diminuée. Des pratiques de formations ont été testées (Brissaud, Cogis \& Péret, 2012). Certes, elles demandent du temps. Qu'est le cout de cette formation comparé à celui de l'arrivée sur le marché du travail de jeunes de plus en plus nombreux maitrisant mal le français? La balle n'est plus dans le camp des linguistes ni des didacticiens.

\section{RÉFÉRENCES BIBLIOGRAPHIQUES}

ACAdÉmie FrançaIse. (1932). Grammaire de l'Académie française. Paris : Firmin-Didot.

Arnauld, A. \& Nicole, P. (1660). Grammaire générale et raisonnée. Paris : Pierre Le Petit.

Bescherelle, L.-N. \& Bescherelle, H. (1834). Grammaire nationale. Paris : Bourgeois-Maze.

Bescherelle, L.-N. \& Bescherelle, H. (1839). Grammaire des épiciers. Paris : Bourgeois-Maze. 
Bescherelle, L.-N. \& Bescherelle, H. (1842). Dictionnaire usuel de tous les verbes français. Paris : Breteau et Pichery.

Bescherelle, L.-N. \& Bescherelle, H. (1843). Le Véritable Manuel des conjugaisons, ou la Science des conjugaisons mise à la portée de tout le monde. Paris : dépôt central des publications classiques.

Bescherelle, L.-N. (1851). Plus de grammaire, ou simples règles d'orthographe, de grammaire, de syntaxe et de prononciation, Manuel usuel et populaire. Tours : Mme Royer.

Bescherelle, L.-N. \& Bescherelle, H. (1884). Le véritable Manuel des conjugaisons ou Dictionnaire des 8000 verbes. Paris : Dentu ( $7^{\mathrm{e}}$ éd.).

Bescherelle. (1950). L'Art de conjuguer ou les 8000 verbes, modèles de conjugaison pour tous les verbes français. Paris: J. Gibert (nouvelle édition).

BesCherelle. (1980). L'Art de conjuguer, dictionnaire de 12000 verbes. Paris : Hatier (nouvelle édition).

Bescherelle. (1999). La conjugaison 12000 verbes. Paris : Hatier (nouvelle édition).

Bescherelle. (2012). La conjugaison pour tous. Tous les verbes français, les tableaux modèles, les règles de conjugaison, les règles d'accord. Paris : Hatier (nouvelle édition).

Benveniste, É, (1965). «Être» et «Avoir» dans leurs fonctions linguistiques. Problèmes de linguistique générale, 1 (chapitre XVI, p. 187-207). Paris : Gallimard.

Blanche-Benveniste, C., (1988). Relation entre deux formes verbales. Pronom et syntaxe. L'approche pronominale et son application au français. Paris : SELAF, 161-202.

Brissaud, C., Chevrot, J.-P. \& Lefrançois, P. (2006). Les formes verbales en /E/ entre 8 et 15 ans : contraintes et conflits dans la construction de savoirs sur une difficulté orthographique majeure du français. Langue française, 151, 74-93.

Brissaud, C., Cogis, D. \& Péret, C., (2012). L'enseignement de l'orthographe : une mission encore possible? L'orthographe en quatre temps. Paris : Champion.

Brunot, F. (1922). La pensée et la langue. Paris, Masson \& C C

Carlotti, A. (2011), Phrase, énoncé, texte, discours, de la linguistique universitaire à la grammaire scolaire. Limoges : Lambert Lucas.

Chervel, A. (1977). Et il fallut apprendre à écrire à tous les petits Français : histoire de la grammaire scolaire. Paris : Payot.

Damourette, J. \& Pichon, F. (1911-1936). Des mots à la pensée. Essai de grammaire de la langue française. Paris : Éditions d'Arthrey.

Élalouf, M.-L. \& Péret, C. (2009). Pratiques d'observation de la langue en France : quelles évolutions? quels obstacles? Dans J. Dolz \& Cl. 
Simard (dir.), Pratiques d'enseignement grammatical (p. 49-72). Québec : Presses de l'Université Laval.

Fleury, S. \& Branca-Rosoff, S. (2010). Une expérience de collaboration entre linguiste et spécialiste de TAL : L'exploitation du corpus CFFP 2000 en vue d'un travail sur l'alternance futur simple/futur périphrastique. Cahiers AFLS, 16 (1), 63-98.

Genouvrier, É. \& Peytard, J., (1970). Linguistique et enseignement du français, Paris : Larousse.

Genouvrier, É. et Gruwez, C., (1972). Grammaire nouvelle pour le CE1. Paris : Larousse.

Gougenheim, G. (1929). Études sur les périphrases verbales de la langue française. Paris : Les Belles lettres.

Gross, M., (1986). Grammaire transformationnelle du français. 1. Syntaxe du verbe. Paris : Malakoff Cantilène.

JEANJEAN, C., (1988). Le futur simple et le futur périphrastique en français parlé, étude distributionnelle. Dans C. Blanche-Benveniste, A. Chervel \& M. Gross (dir.), Grammaire et histoire de la grammaire : hommage à la mémoire de Jean Stefanini (p. 235-257). Publication de l'université de Provence.

JönSSON, A. (2010). Incrémentalisme. Dictionnaire des politiques publiques, 317-325.

Holtz, L., (2010). Donat et la tradition de l'enseignement grammatical. Paris : CNRS Éditions

Lindblom, C. (1959). The Science of "Muddling Through". Public Administration Review, 19 (2), 79-88.

Lhomond, C. F. (1850). Éléments de la grammaire latine (2e édition de l'édition de 1779). Paris : Belin.

Lhomond, C. F. (1780). Élémens de la grammaire françoise. Paris : Colas. Maupas, C. (1632). Grammaire et syntaxe françoise. Rouen : Cailloué.

Ministère DE L'ÉduCATION NATIONALE. (1972). Instructions relatives à l'enseignement du français à l'école élémentaire. Bulletin Officiel de l'Éducation nationale, circulaire 72-474 du 4 décembre 1972.

Ministère DE L'ÉduCATION NATIONALE. (2002). Horaires et programmes d'enseignement de l'école primaire. Bulletin officiel de l'Éducation nationale, hors-série $\mathrm{n}^{\mathrm{o}} 1$.

Ministère DE L'ÉduCATion nATIONALE. (2008), Horaires et programmes d'enseignement de l'école primaire. Bulletin officiel de l'Éducation nationale, hors-série $\mathrm{n}^{\circ} 3$.

Ministère de L'INSTRUCTION PUBLIQUE. (1923), Instructions relatives au nouveau plan d'études des écoles primaires élémentaires.

PÉret, C. \& SAutot, J.-P. (2012a). Le verbe : entre curriculum institué et curriculum réel. Dyptique, 23, Actes du XI' congrès de l'AiRDF, 253274 . 
Péret, C. \& SAUtot, J.-P. (2012b). Le verbe auxiliaire : état d'une notion chez les élèves. Communication présentée au colloque Le complexe $d u$ verbe, Lyon 30 mai 2012.

Péret, C., Sautot, J.-P. \& Brissaud, C. (2008). Les professeurs entrant dans le métier et la norme orthographique. Dans C. Brissaud, J.-P. Jaffré \& J.-C. Pellat (dir.), Nouvelles recherches en orthographe (p. 203-214), Actes des journées d'études des 14 et 15 juin 2007, Université de Strasbourg. Limoges : Lambert Lucas.

Rouchette, M., (1971). Plan de rénovation de l'enseignement du français à l'école élémentaire. Recherches pédagogiques, 47.

SAINT-GÉRAND, J.-P. (2000). Sentiment national, constructions et représentations linguistiques au XIX ${ }^{\mathrm{e}}$ siècle. Les travaux des frères Bescherelle à l'aune du concept de «français de référence». Cahiers de l'Institut de linguistique de Louvain, 26 (1-4), 55-73. 